PROCEEDINGS OF THE

AMERICAN MATHEMATICAL SOCIETY

Volume 128 , Number 10, Pages 3125-3128

S 0002-9939(00)05390-9

Article electronically published on April 28, 2000

\title{
NONCOMPLEX SMOOTH 4-MANIFOLDS WITH GENUS-2 LEFSCHETZ FIBRATIONS
}

\author{
BURAK OZBAGCI AND ANDRÁS I. STIPSICZ
}

(Communicated by Ronald A. Fintushel)

\begin{abstract}
We construct noncomplex smooth 4-manifolds which admit genus2 Lefschetz fibrations over $S^{2}$. The fibrations are necessarily hyperelliptic, and the resulting 4-manifolds are not even homotopy equivalent to complex surfaces. Furthermore, these examples show that fiber sums of holomorphic Lefschetz fibrations do not necessarily admit complex structures.
\end{abstract}

In this paper we will prove the following theorem.

Theorem 1.1. There are infinitely many (pairwise nonhomeomorphic) 4-manifolds which admit genus-2 Lefschetz fibrations but do not carry complex structure with either orientation.

Matsumoto [6] showed that $S^{2} \times T^{2} \# 4 \overline{\mathbb{C} P^{2}}$ admits a genus-2 Lefschetz fibration over $S^{2}$ with global monodromy $\left(\beta_{1}, \ldots, \beta_{4}\right)^{2}$, where $\beta_{1}, \ldots, \beta_{4}$ are the curves indicated by Figure 1 (For definitions and details regarding Lefschetz fibrations see 6], 5].)

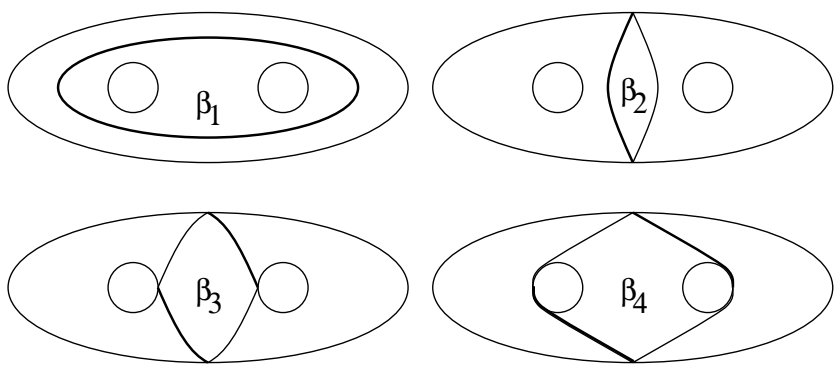

FIGURE 1.

Let $B_{n}$ denote the smooth 4-manifold which admits a genus-2 Lefschetz fibration over $S^{2}$ with global monodromy

$$
\left(\left(\beta_{1}, \ldots, \beta_{4}\right)^{2},\left(h^{n}\left(\beta_{1}\right), \ldots, h^{n}\left(\beta_{4}\right)\right)^{2}\right)
$$

where $h=D\left(a_{2}\right)$ is a positive Dehn twist about the curve $a_{2}$ indicated in Figure 2

Received by the editors October 13, 1998 and, in revised form, November 24, 1998.

2000 Mathematics Subject Classification. Primary 57R55; Secondary 57R65, 57M50.

Key words and phrases. Lefschetz fibrations, 4-manifolds, complex structures.

(C)2000 American Mathematical Society 
Theorem 1.2. For the 4-manifold $B_{n}$ given above we have $\pi_{1}\left(B_{n}\right)=\mathbb{Z} \oplus \mathbb{Z}_{n}$.

Proof. Standard theory of Lefschetz fibrations gives that

$$
\pi_{1}\left(B_{n}\right)=\pi_{1}\left(\Sigma_{2}\right) /\left\langle\beta_{1}, \ldots, \beta_{4}, h^{n}\left(\beta_{1}\right), \ldots, h^{n}\left(\beta_{4}\right)\right\rangle .
$$

Let $\left\{a_{1}, b_{1}, a_{2}, b_{2}\right\}$ be the standard generators for $\pi_{1}\left(\Sigma_{2}\right)$ (Figure 2).

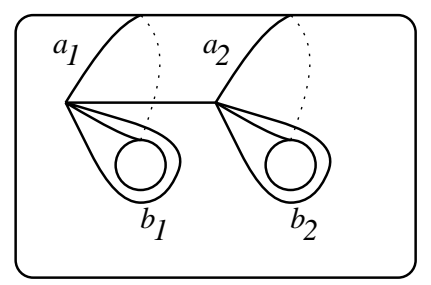

FiguRE 2.

Then we observe that

$\beta_{1}=b_{1} b_{2}$,

$\beta_{2}=a_{1} b_{1} a_{1}^{-1} b_{1}^{-1}=a_{2} b_{2} a_{2}^{-1} b_{2}^{-1}$

$\beta_{3}=b_{2} a_{2} b_{2}^{-1} a_{1}$

$\beta_{4}=b_{2} a_{2} a_{1} b_{1}$

$h^{n}\left(\beta_{1}\right)=b_{1} b_{2} a_{2}^{n}$,

$h^{n}\left(\beta_{2}\right)=\beta_{2}$,

$h^{n}\left(\beta_{3}\right)=\beta_{3}$,

$h^{n}\left(\beta_{4}\right)=b_{2} a_{2}^{n+1} a_{1} b_{1}$.

Hence

$$
\begin{aligned}
\pi_{1}\left(B_{n}\right) & =\left\langle a_{1}, b_{1}, a_{2}, b_{2} \mid b_{1} b_{2},\left[a_{1}, b_{1}\right], b_{2} a_{2} b_{2}^{-1} a_{1}, b_{2} a_{2} a_{1} b_{1}, b_{1} b_{2} a_{2}^{n}, b_{2} a_{2}^{n+1} a_{1} b_{1}\right\rangle \\
& =\left\langle a_{2}, b_{2} \mid\left[a_{2}, b_{2}\right], a_{2}^{n}\right\rangle \\
& =\mathbb{Z} \oplus \mathbb{Z}_{n},
\end{aligned}
$$

and this concludes the proof.

The above definition of $B_{n}$ provides a handlebody decomposition for it [5] and shows, in particular, that the Euler characteristic $\chi\left(B_{n}\right)$ is equal to 12 . Since $B_{n}$ is the fiber sum of two copies of $S^{2} \times T^{2} \# 4 \overline{\mathbb{C} P^{2}}$, we get that the signature $\sigma\left(B_{n}\right)=-8$. Consequently, $b_{2}\left(B_{n}\right)=12$ and $b_{2}^{+}\left(B_{n}\right)=2, b_{2}^{-}\left(B_{n}\right)=10$. Let $M_{n}$ deonote the $n$-fold cover of $B_{n}$ with $\pi_{1}\left(M_{n}\right) \cong \mathbb{Z}$. Easy computation shows that $b_{2}^{+}\left(M_{n}\right)=2 n$ and $b_{2}^{-}\left(M_{n}\right)=10 n$.

Theorem 1.3. $B_{n}$ does not admit a complex structure.

Proof. Assume that $B_{n}$ admits a complex structure and let $M_{n}^{\prime}$ denote the minimal model of $M_{n}$. By the Enriques-Kodaira classification of complex surfaces [1] (since $b_{1}\left(M_{n}^{\prime}\right)=1$ ), $M_{n}^{\prime}$ is either a surface of class VII (in which case $b_{2}^{+}\left(M_{n}^{\prime}\right)=0$ ), a secondary Kodaira surface (in which case $b_{2}\left(M_{n}^{\prime}\right)=0$ ) or a (minimal) properly elliptic surface. Since $b_{2}^{+}\left(M_{n}^{\prime}\right)=b_{2}^{+}\left(M_{n}\right)=2 n$, the first two possibilities are ruled out. 
Suppose now that $M_{n}^{\prime}$ admits an elliptic fibration over a Riemann surface. If the Euler characteristic of $M_{n}^{\prime}$ is 0 , then (following from the fact that $b_{1}\left(M_{n}^{\prime}\right)=$ $b_{3}\left(M_{n}^{\prime}\right)=1$ ) we get that $b_{2}\left(M_{n}^{\prime}\right)=0$, which leads to the above contradiction. Suppose finally that $M_{n}^{\prime}$ is a minimal elliptic surface with positive Euler characteristic. Since $b_{1}\left(M_{n}^{\prime}\right)=1$, it can only be fibered over $S^{2}$ (see for example 2] ). In that case (according to 4], for example) its fundamental group is

$$
\pi_{1}\left(M_{n}^{\prime}\right)=\left\langle x_{1}, \ldots x_{k} \mid x_{i}^{p_{i}}=1, i=1, \ldots, k ; x_{1} \cdots x_{k}=1\right\rangle .
$$

This cannot be isomorphic to $\mathbb{Z}$, since if $\pi_{1}\left(M_{n}\right) \cong \mathbb{Z}=\langle a\rangle$, then $x_{1}=a^{m_{1}}$ for some $m_{1} \in \mathbb{Z}$, so $a$ has finite order, which is a contradiction. Consequently the assumption that $B_{n}$ is complex leads us to a contradiction; hence the theorem is proved.

Remark 1.4. The above proof, in fact, shows that $B_{n}$ is not even homotopy equivalent to a complex surface - our arguments used only homotopic invariants (the fundamental group, $b_{2}$ and $b_{2}^{+}$) of the 4-manifold $B_{n}$. Note that the same idea shows that $\bar{B}_{n}$ (the manifold $B_{n}$ with the opposite orientation) carries no complex structure: The arguments involving the fundamental group, $b_{2}$ and the Euler characteristic only, apply without change. Since $b_{2}^{+}\left(\bar{M}_{n}\right)=b_{2}^{-}\left(M_{n}\right)=10 n \neq 0$, the arguments using that fact that $b_{2}^{+} \neq 0$ apply as well.

Proof of Theorem 1.1. By the definition of the 4-manifolds $B_{n}$ we get infinitely many manifolds admitting genus-2 (consequently hyperelliptic) Lefschetz fibrations which are (by Theorem 1.2) nonhomeomorphic. As Theorem 1.3 and the above remark show, the manifolds $B_{n}$ do not carry complex structures with either orientation; hence the proof of the Theorem 1.1 is complete.

Remark 1.5. We would like to point out that similar examples have been found by Fintushel and Stern [3] - they used Seiberg-Witten theory to prove that their (simply connected) Lefschetz fibrations are noncomplex.

Note that $B_{n}$ is given as the fiber sum of two copies of $S^{2} \times T^{2} \# 4 \overline{\mathbb{C} P^{2}}$, hence provides an example of the phenomenon that the fiber sum of holomorphic Lefschetz fibrations is not necessarily complex.

\section{ACKNOWLEDGEMENT}

Examples of genus-2 Lefschetz fibrations with $\pi_{1}=\mathbb{Z} \oplus \mathbb{Z}_{n}$ were also constructed (as fiber sums) independently by Ivan Smith [7].

\section{REFERENCES}

1. W. Barth, C. Peters and A. Van de Ven, Compact complex surfaces, Springer-Verlag, 1984. MR 86c:32026

2. R. Friedman and J. Morgan, Smooth four-manifolds and complex surfaces, Springer-Verlag, 1994. MR 95m:57046

3. R. Fintushel and R. Stern, Private communication.

4. R. Gompf, Nuclei of elliptic surfaces, Topology 30 (1991), pp. 479-511. MR 92f:57042

5. R. Gompf and A. Stipsicz, An introduction to 4-manifolds and Kirby calculus, AMS Graduate Studies in Math., vol. 20, 1999. 
6. Y. Matsumoto, Lefschetz fibrations of genus two - a topological approach, Proceedings of the 37th Taniguchi Symposium on Topology and Teichmüller Spaces, ed. Sadayoshi Kojima et al., World Scientific (1996), pp. 123-148. CMP 99:06

7. I. Smith, Symplectic geometry of Lefschetz fibrations, Dissertation, Oxford, 1998.

Department of Mathematics, University of California Irvine, Irvine, California 92697

E-mail address: bozbagci@math.uci.edu

Current address: Department of Mathematics, Michigan State University, East Lansing, Michigan 48824

E-mail address: bozbagci@math.msu.edu

Department of Analysis, Elte TTK, Múzeum krt. 6-8, Budapest, Hungary

E-mail address: stipsicz@cs.elte.hu 